\title{
A New Precipitation Prediction Method Based on CEEMDAN-IOWA-BP Coupling
}

Fuping LIU ( $\sim$ Liufp@stu.ncwu.edu.cn )

North-China University of Water Conservancy and Electric Power: North China University of Water Resources and Electric Power https://orcid.org/0000-0001-8669-1161

Ying Liu

North China University of Water Resources and Electric Power

Chen Yang

North China University of Water Resources and Electric Power

Ruixun Lai

Yellow River Institute of Hydraulic Research

\section{Research Article}

Keywords: precipitation prediction, CEEMDAN, IWOA-BP, chaos mapping, roulette algorithm

Posted Date: February 22nd, 2022

DOI: https://doi.org/10.21203/rs.3.rs-1361955/v1

License: (1) (1) This work is licensed under a Creative Commons Attribution 4.0 International License.

Read Full License 


\title{
A new precipitation prediction method based on CEEMDAN-IOWA-BP coupling
}

\author{
Fuping Liu ${ }^{a}$, Ying Liu ${ }^{a}$, Chen Yang ${ }^{a, *}$, Ruixun Lai ${ }^{\mathrm{b}, *}$ \\ ${ }^{\text {a }}$ School of Water Conservancy, North China University of Water Resources and Electric \\ Power, Zhengzhou, China, 450046 \\ ${ }^{b}$ Yellow River Institute of Hydraulic Research, Zhengzhou, China, 450003 \\ Corresponding autor:suberliu@163.com, liuncwu@163.com
}

\begin{abstract}
Precipitation is the most basic part of the water cycle process. Aiming at the problem of low prediction accuracy caused by the nonlinear and unstable characteristics of the precipitation series, a new precipitation prediction method based on the
\end{abstract} CEEMDAN-IWOA-BP coupling model is proposed. This method first uses the complete ensemble empirical mode decomposition with adaptive noise (CEEMDAN) to decompose the original precipitation sequence, and obtains a series of intrinsic mode function (IMF) and residual terms (Res) as inherent potential influencing factors, innovatively introduce TENT chaotic mapping and roulette algorithm to improve the Whale Optimization Algorithm (WOA), use IMFs and Res as the input of the Improve Whale Optimization Algorithm (IWOA) to optimize Back Propagation (BP) neural network prediction model, and finally superimpose the predicted values as ultima result. The present method was applied to predict the annual precipitation from 1958 to 2017 in Sichuan Province.Compared with the prediction results of other models, the CEEMDAN-IWOA-BP coupled model has significantly improved prediction accuracy than the single model, and the prediction error index of the Back Propagation(BP) neural network optimized by the Genetic Algorithm (GA) and Particle Swarm 
Optimization (PSO) algorithms is smaller, and the optimization accuracy and solving ability are significantly enhanced compared with the unimproved WOA.

Keywords: precipitation prediction; CEEMDAN; IWOA-BP; chaos mapping; roulette algorithm

\section{Introduction}

The issue of water resources is one of the important scientific issues that the scientific community attaches importance to (Xia et al. 2011), and precipitation is the most basic link in the cycle of water resources (Yang 2020). For many years, the forecast of mid- and long-term precipitation has mainly adopted statistical forecasting methods, which include two types of time series analysis and multivariate analysis (Lei 2020). However, the statistical analysis model lacks adaptability, which makes it difficult to update the model itself. At the same time, the uncertainty of the model also causes the instability of prediction and inspection accuracy (Xu et al. 2006). Therefore, there is an urgent need for more effective forecasting methods.

Since David et al (1986). proposed the BP neural network model, the crossdiscipline of machine learning has gradually been applied to wind power load (Wang and Sun, 2015), operation and maintenance (Peng and Liu, 2014), energy supply and demand (Xie et al. 2019), news media (Cao et al. 2014), complex systems (Zhao et al. 2016), land and resources (Han et al. 2015) and meteorological analysis (Ren et al. 2014) and other key factors forecasting in multiple industries. Among them, many scholars have made many achievements in the field of precipitation prediction in the field of meteorological analysis (Shen et al. 2020, Jia et al. 2013, Sun and Lin 2003). As the 
requirements for model solving capabilities increase, coupled algorithms for prediction have begun to be adopted by scholars. Ahmed et al. (2011) proposed a combination of Genetic Algorithm (GA) and Probabilistic Neural Network (PNN) to improve the prediction accuracy of PNN. Bo et al. (2005) proposed the combination of Support Vector Machine (SVM) and Artificial Neural Network (ANN) to improve the reliability of the prediction model. However, due to the shortcomings of neural network algorithms, it is often used in combination with optimization algorithms (Wang et al. 2017, Hu et al. 2016, Yu et al. 2004). The commonly used optimization algorithms are Genetic Algorithm (Gou et al. 2019), Particle Swarm Optimization algorithm (Qiu er al. 2017), Simulated Degradation Algorithm (Jin and Teng 2013), Ant Colony Algorithm (Li 2018) and Whale Optimization Algorithm (Long et al. 2017).

Compared with other algorithms, the Whale Optimization Algorithm has the advantages of simple structure, fewer parameters, and faster iteration speed. This article uses the whale optimization algorithm to build the model. In view of the disadvantages of the traditional whale algorithm such as weak global search ability, the TENT chaotic map is introduced to the population Initialization, to further improve the global search capability of the algorithm, and introduce the roulette algorithm to enhance the local optimization capability of the algorithm as IWOA.

In practical applications, the coupling model uses various decomposition methods to combine with different prediction models. Based on the decomposition algorithm, the data is preprocessed to reduce the non-stationarity of the sequence to achieve the purpose of improving the accuracy of the model (Xu et al. 2021). In this paper, the items 
decomposed by CEEMDAN are used as the input of the IWOA-BP prediction model, and the weights and thresholds of the BP neural network are optimized through IWOA to improve the robustness and accuracy of the BP neural network, then superimpose the output of the BP neural network as the prediction result. Finally, the model is applied to the measured precipitation sequence, and the superiority of the CEEMDAN-IWOABP prediction model is verified through the prediction fitting of different models and the comparison of multiple error indicators.

\section{CEEMDAN Decomposition}

CEEMDAN decomposition is an auxiliary data analysis method proposed by Torres M E (2011). The specific steps of the CEEMDAN method can be found in reference $\mathrm{Li}$ and $\mathrm{Li}$ (2015). For the traditional EMD decomposition (Huang et al. 1998), there is modal aliasing phenomenon and the residual white noise in the decomposition process of EEMD (Wu and Huang 2009). The CEEMDAN algorithm adds an adaptive white noise sequence at each data decomposition stage, which effectively alleviates the phenomenon of modal aliasing, and eliminates the effect of artificial white noise on the original sequence, which reduces the data reconstruction error and improves the completeness of the data decomposition. In the decomposition process, due to the different contributions of IMFs and Res to the time series, IMFs and Res can be used as time driving factors, and the prediction of the data series is considered to be the prediction of the two parts of IMFs and Res. When the sequence reaches the termination condition of decomposition, $\mathrm{K}$ modal components are finally obtained, and the original sequence can be decomposed into 


$$
X(t)=R(t)+\sum_{k=1}^{K} I M F_{k}
$$

Where $\mathrm{X}(\mathrm{t})$ is the original sequence, $\mathrm{R}(\mathrm{t})$ is the residual term, and $\mathrm{IMF}_{\mathrm{k}}$ is the internal mode component of the decomposed series. The final sequence is decomposed by CEEMDAN to obtain a series of IMFs ranging from high frequency to low frequency, and the original sequence can be accurately and completely reconstructed by using the characteristics of noise adaptation.

\section{BP Neural Network Based on IWOA Optimization}

\subsection{Basic WOA Algorithm}

The Whale Optimization Algorithm is an intelligent optimization algorithm proposed by Mirjalili (2016). The proposed algorithm is affected by the predation of humpback whales and imitates the hunting process of whales, as follows.

(1) Search for prey. Whales achieve their hunting goals by updating their positions when searching the space, this behavior is represented by equations (2) and (3).

$$
\begin{gathered}
\vec{D}=\left|\vec{C} \cdot \overrightarrow{X^{*}}(\varphi)-\vec{X}\right| \\
\vec{X}(\varphi+1)=\overrightarrow{X^{*}}(\varphi)-\vec{A} \cdot \vec{D}
\end{gathered}
$$

Where $\mathrm{D}$ is the distance between the current optimal individual and other individuals; $\varphi$ is the current it eration number; $\overrightarrow{\mathrm{X}^{*}}$ is the vector position of the current optimal individual; $\vec{X}$ is the position vector of other individuals; $\vec{A}$ and $\vec{C}$ is a vector of coefficients, $\vec{A}=2 \vec{a} \cdot \vec{r}-\vec{a}, \vec{C}=2 \vec{r}$, a is a vector that linearly decreases with the number of iterations on $[0,2]$, and $\vec{r}$ is a random vector on $[0,1]$.

(2) Surround and attack. This process includes two local optimization behaviors, the shrinking enveloping mechanism and the spiral update position, as shown in Figures 
1 and 2.
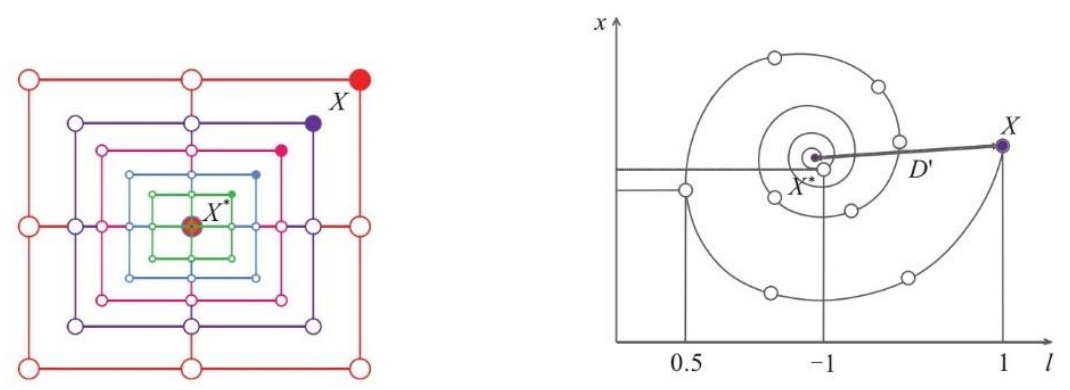

Fig. 1 Shrinking and enveloping mechanism; Fig. 2 Spiral update position

Among them, the shrinking and enclosing mechanism is realized by reducing $\vec{a}$, and the range of $\overrightarrow{\mathrm{A}}$ is $[-\mathrm{a}, \mathrm{a}]$. As it approaches the optimal individual, the whale will surround its prey in a spiral manner. This behavior is described below.

$$
\vec{X}(\varphi+1)=\overrightarrow{D^{\prime}} e^{m l} \cos (2 \pi l)+\overrightarrow{X^{*}}(\varphi)
$$

Where $\overrightarrow{\mathrm{D}^{\prime}}$ is the distance between the best position and the current optimal solution; $m$ is the shape constant of the spiral; 1 is a random number between $[-1,1]$. When whales are hunting, there is a $50 \%$ probability of choosing the above two hunting methods. The behavior is described as follows.

$$
\vec{X}(\varphi+1)=\left\{\begin{array}{c}
\overrightarrow{X^{*}}(\varphi)-\vec{A} \cdot \vec{D}, \quad p \leq 0.5 \\
\overrightarrow{D^{\prime}} e^{m l} \cos (2 \pi l)+\overrightarrow{X^{*}}(\varphi), \quad p>0.5
\end{array}\right.
$$

(3) Simulate predation. When $|A|<1$, the position of the optimal individual is the position of the prey, and other individuals in the group will adjust their positions to hunt according to the optimal individual; when $|A| \geq 1$, the group is forced to update the position information of randomly selected individuals Until the optimal solution is obtained, the process is described as follows.

$$
\begin{gathered}
\overrightarrow{D^{\prime \prime}}=\left|\vec{C} \cdot \vec{X}_{\text {rand }}-\vec{X}\right| \\
\overrightarrow{X_{z}}(\varphi+1)=\vec{X}_{\text {rand }}-\vec{A} \cdot \overrightarrow{D^{\prime \prime}}
\end{gathered}
$$


Where $\vec{X}_{\text {rand }}$ is the position vector of randomly selected individuals in the group.

\subsection{Improved WOA Algorithm}

The basic WOA has shortcomings such as weak global search ability and easy to fall into local optimum, slow local optimization speed and so on. The traversal of the TENT chaotic map has uniformity and randomness, which can make the algorithm easy to escape from the local optimal solution, thereby maintaining the diversity of the population and improving the global search ability. This paper first uses TENT chaotic mapping to initialize the population, which greatly eliminates the randomness of the initial population, makes the initial population more evenly distributed within the parameter range, and further improves the global search ability of the WOA. The TENT chaotic map is as follows.

$$
X_{t+1}= \begin{cases}\frac{X_{t}}{\alpha} & , X_{t} \in[0, \alpha) \\ \frac{1-X_{t}}{1-\alpha} & , X_{t} \in[\alpha, 1]\end{cases}
$$

In the formula, $\alpha \in(0,1)$, when $\alpha=0.5$, the system will present a short period state and not belong to a chaotic system. In this study, the value of $\alpha$ is 0.6 .

The basic WOA is blind in the process of optimizing and predation, and cannot fully integrate the iterative experience to update the population. Secondly, this paper uses the roulette algorithm to select prey. Under the premise of ensuring the global development capability, it can rely on the current iteration results to select more representative individuals, speed up the search convergence speed, and then quickly find the local optimization. The basic idea of the roulette algorithm is that the probability of an individual being selected is directly proportional to the value of its fitness function. The core of the algorithm lies in the probability of individual selection, 
the cumulative probability and how to select individuals. The individual probability and cumulative probability are expressed as follows.

$$
\begin{gathered}
P\left(x_{i}\right)=\frac{f\left(x_{i}\right)}{\sum_{j=1}^{N} f\left(x_{j}\right)} \\
Q\left(x_{i}\right)=\sum_{k=1}^{i} P\left(x_{k}\right)
\end{gathered}
$$

In the formula, $x_{i}$ is the individual and $\mathrm{N}$ is the total. The implementation steps of the roulette algorithm: initialize the fitness of the individual; calculate the individual selection probability and cumulative probability of each individual; generate a random number $x_{\text {rand }}$ in the interval $[0,1]$ and compare it with the cumulative probability $Q\left(x_{i}\right)$, if $x_{\text {rand }}<Q\left(x_{i}\right)$, then select this individual $x_{i}$.

To test the optimization level of IWOA compared to traditional WOA, the benchmark test function Sphere is selected for verification, where the population size is 30 , the maximum number of iterations is 1000 , and the number of tests is 30 , the schematic diagram of the optimization process and the convergence curve are shown in Figure 3. It can be seen from the comparison that the convergence speed, optimization accuracy and global search ability of IWOA are stronger than traditional WOA, and traditional WOA falls into a local optimal solution dilemma in the later stage, and the IWOA avoids this problem well. In general, IWOA has higher solving ability and optimization precision, which verifies the superiority of the improved method. 

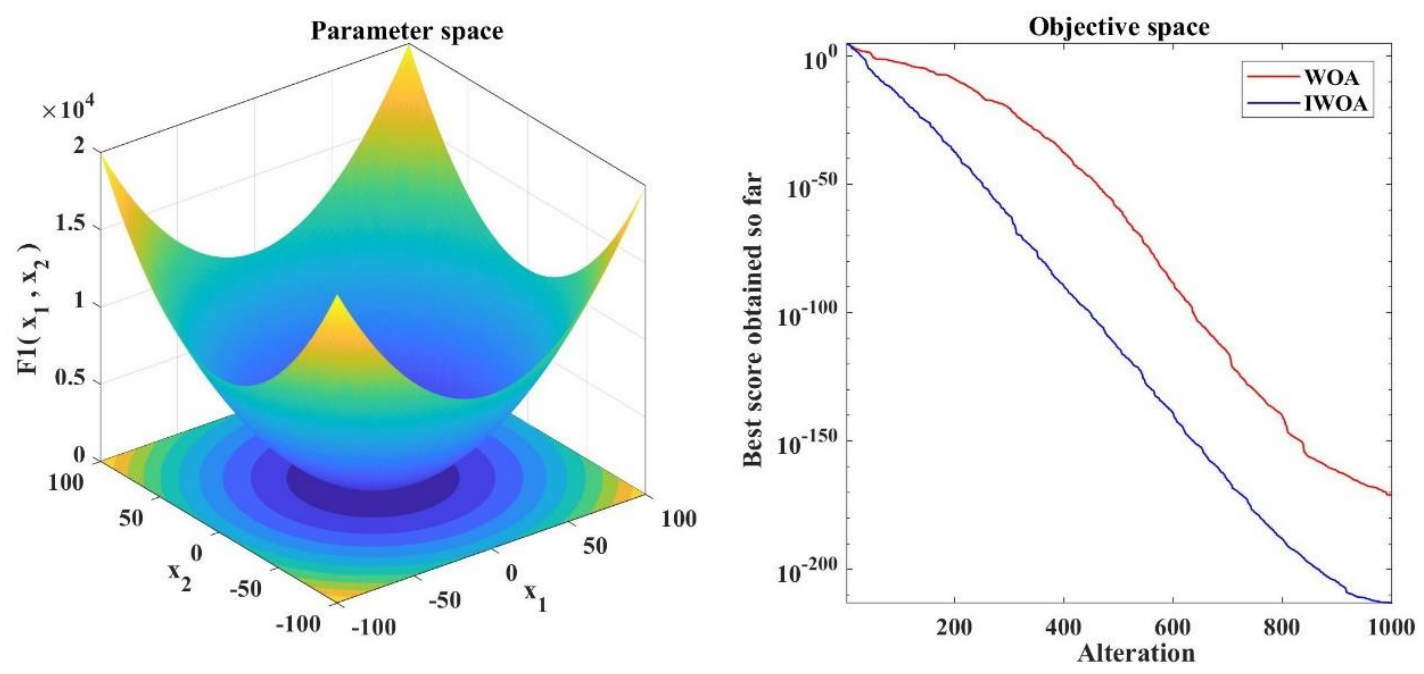

Fig. 3 Optimization process and convergence curve

\subsection{Improved WOA Optimized BP Neural Network}

BP neural network is a multi-layer feedforward neural system. Its main characteristics are signal propagates forward and error propagates backward. BP neural network has self-learning ability and nonlinear function mapping ability. The structure of the three-layer BP neural network is shown in Figure 4.

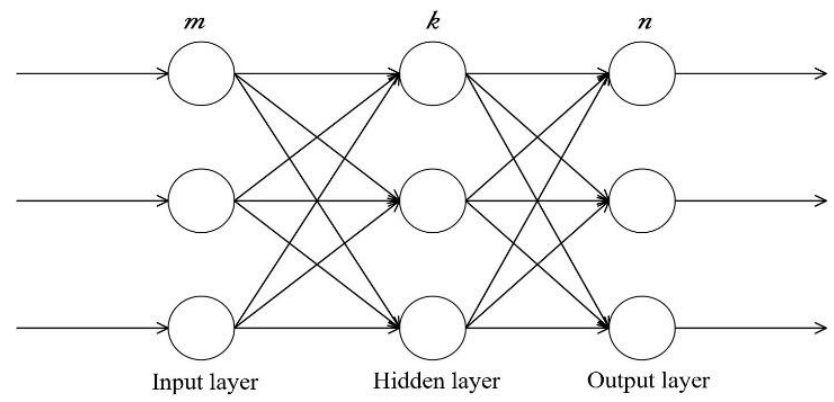

Fig. 4 Topological structure of three-layer BP neural network

The activation function of the input layer of the BP neural network in the model is a sigmoid function, the input samples are normalized by the mapminmax function, the transfer function of the hidden layer is a continuous differentiable tasing function, and the output layer is a linear function purelin. The IWOA optimizes the initial weights and thresholds of the BP neural network, thereby speeding up the iteration speed of the 
BP neural network, and enhancing the optimization ability and robustness of the model.

The operation flow of BP neural network optimized by IWOA based on CEEMDAN decomposition is shown in Figure 5.

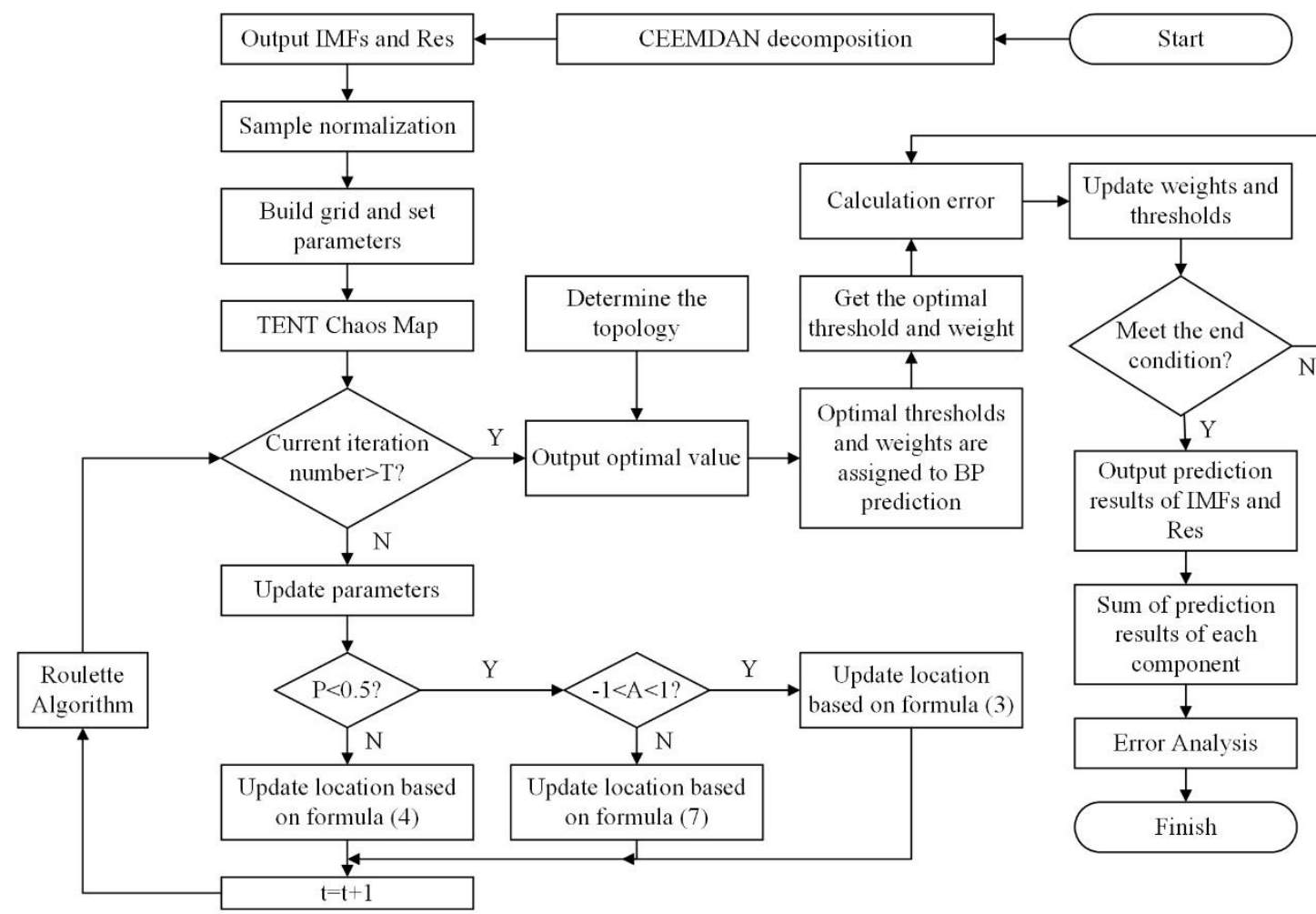

Fig. 5 Forecast flow chart based on CEEMDAN-IWOA-BP

\section{Example Application}

Sichuan Province is located in the inland of southwest China, with complex topography and large differences in topography, the topography is high in the west and low in the east, affected by the monsoon and topography, the annual variation of precipitation varies greatly. A more accurate forecast of annual precipitation will provide corresponding reference value for the protection and management of water resources, water environmental protection and governance, and disaster prevention and mitigation in the province. The measured precipitation sequence of the province from 1958 to 2017 is shown in Figure 6. The sequence is long enough and the data accuracy 
is high, and the representativeness of the data can be guaranteed.

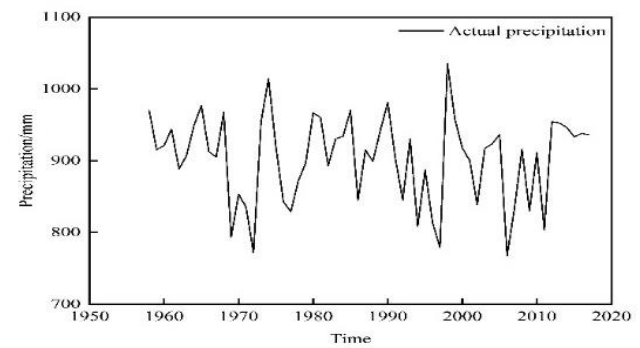

Fig. 6 The precipitation sequence of Sichuan Province from 1958 to 2017

\subsection{CEEMDAN Decomposition of Annual Precipitation Series}

The CEEMDAN method is used to decompose the precipitation sample data of Sichuan Province from 1958 to 2017 to reduce the volatility of the original precipitation series. Set the CEEMDAN algorithm parameters Nstd to 0.2, NE to 500, and Maxlter to 5000 , the original precipitation sequence is finally decomposed into 6 IMF and one Res. The Res indicates that the 60-year precipitation series in Sichuan Province has shown a downward trend as a whole, and the decomposition results are shown in Figure 7.

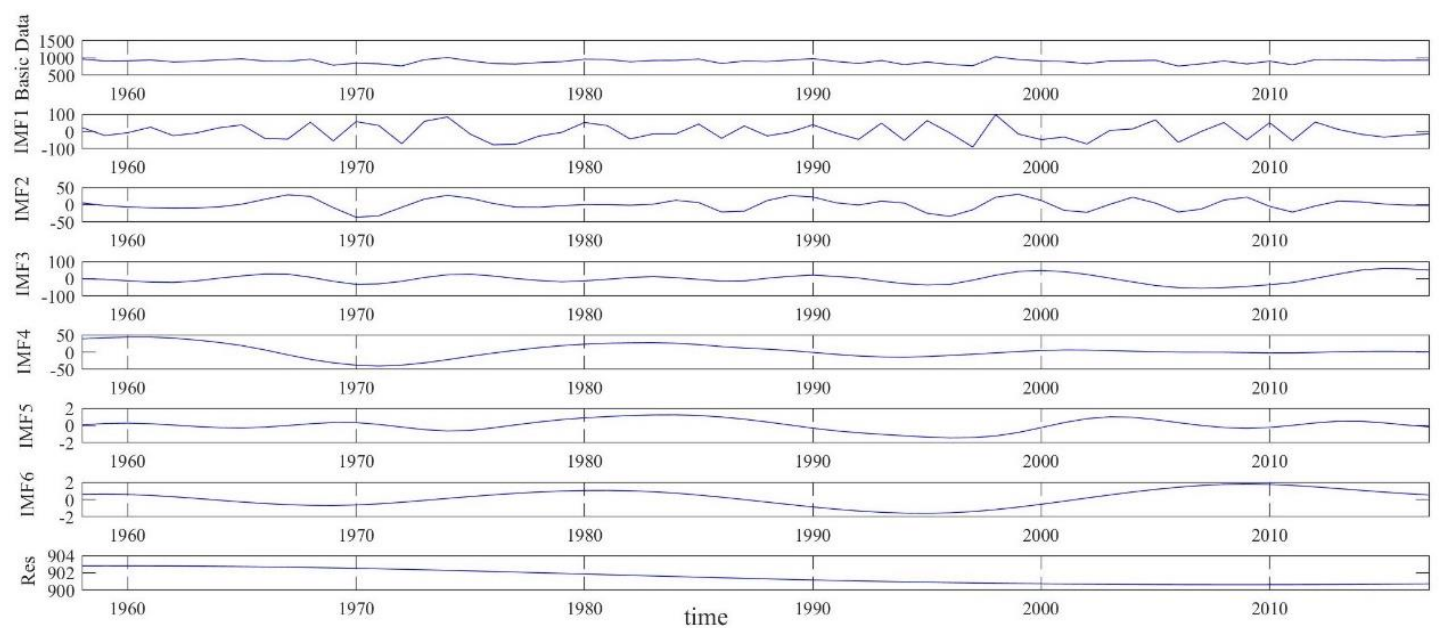

Fig. 7 CEEMDAN decomposition of precipitation series results

\subsection{Model Construction and Prediction}

In order to verify the effectiveness of the model, the improved forecast model's 
annual precipitation forecast results are compared and verified with the traditional BP neural network, the BP neural network optimized by WOA, the BP neural network optimized by GA, and the BP neural network optimized by PSO. The IMF and Res components decomposed by CEEMDAN are used as the input items of the five prediction models, that is, the independent variables, the number of groups is 60 , of which the first 55 groups are the training set, and the last 5 groups are the test set, to verify the prediction effect of the BP neural network optimized by IWOA on precipitation based on existing historical data.

Among them, the parameters of the BP neural network are set as: the number of iterations epo is 100 , the learning rate $l r$ is 0.1 , and the target error rate goal is 0.0001 . The model parameters of IWOA are set as: population size $N$ is 30 , maximum number of iterations $T_{\max }$ is $30, \omega \in[0.4,0.99]$. The IMF and Res decomposed by CEEMDAN are used as the independent variables of the prediction model, the output item is the corresponding prediction value of precipitation, and the prediction value of each item is superimposed as the final prediction value. The prediction results of the five models are compared with the true value as shown in Figure 8. It can be seen from the figure that the prediction result of a single and unoptimized BP neural network is worse than the prediction results of the other four models. The prediction effect of the BP neural network optimized by IWOA on actual precipitation is significantly better than the other four prediction models. 


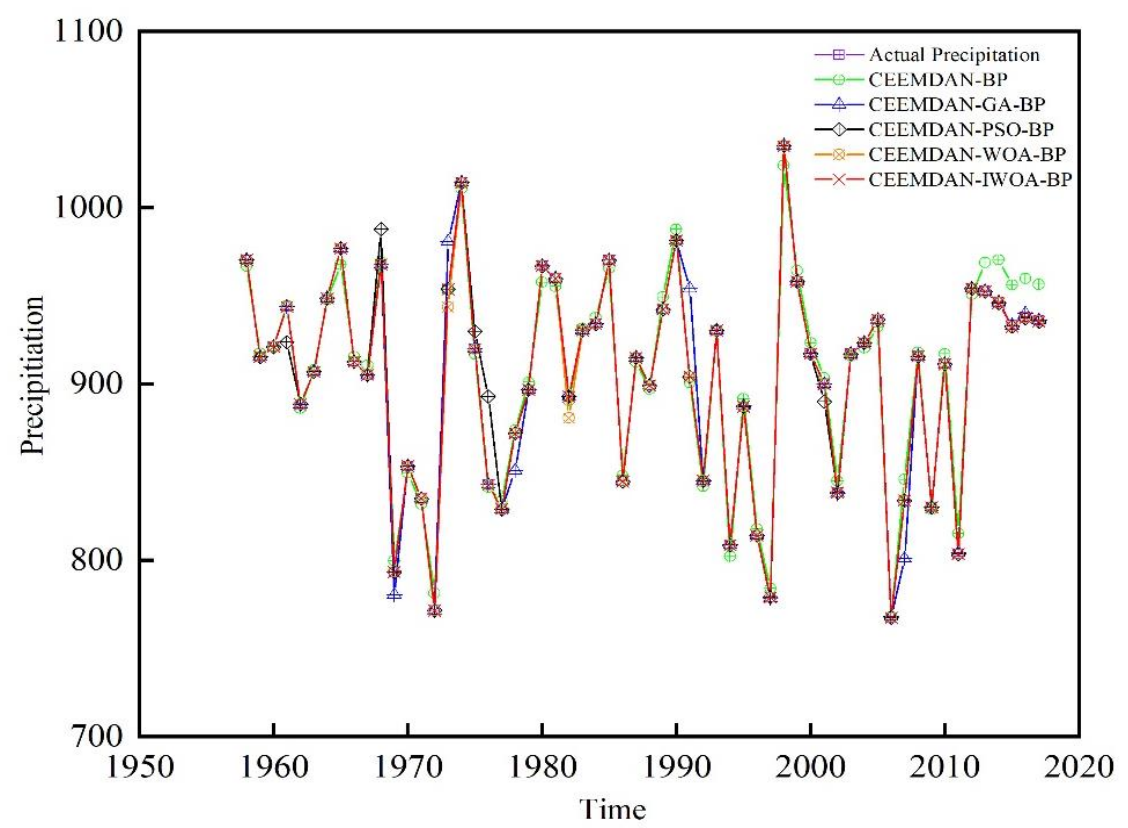

Fig. 8 The prediction results of five different models

\subsection{Error Comparative Analysis}

Based on the same data set, the $\mathrm{R}^{2}$ value, mean square error (MSE), root mean square error (RMSE) and mean absolute percentage error (MAPE) are used as evaluation indicators to evaluate the accuracy of the model, compare and analyze different models. The mathematical expression is as follows.

$$
\begin{gathered}
R^{2}=\frac{\sum_{i=1}^{N}(\hat{y}(t)-\bar{y})^{2}}{\sum_{i=1}^{N}(y(t)-\bar{y})^{2}} \\
M S E=\frac{1}{N} \sum_{i=1}^{N}(\hat{y}(t)-y(t))^{2} \\
R M S E=\sqrt{\frac{1}{N} \sum_{i=1}^{N}(\hat{y}(t)-y(t))^{2}} \\
M A P E=\frac{100 \%}{N} \sum_{i=1}^{N}\left(\frac{\hat{y}(t)-y(t)}{y(t)}\right)
\end{gathered}
$$

Where $\mathrm{N}$ is the number of samples involved in the calculation, $\hat{\mathrm{y}}(\mathrm{t})$ is the predicted value of precipitation in year $t, \bar{y}$ is the average value of the sample, and $y(t)$ is the true value of precipitation in year t. Table 1 shows the evaluation index values of different models, and Figure 8 shows the comparison of the prediction errors 
of different models.

It can be seen from Table 1 and Figure 9 that the $\mathrm{R}^{2}$ value of the CEEMDANIWOA-BP model is closest to 1 , and the degree of linear fit is better than the other four prediction models, the error evaluation indexes MAPE, MSE and RMSE are 0.014\%, $9.344 \mathrm{~mm}^{2}$ and $2.061 \mathrm{~mm}$ respectively, this result shows that the CEEMDAN-IWOABP model has higher precipitation prediction accuracy. Compared with the four models of CEEMDAN-BP, CEEMDAN-GA-BP, CEEMDAN-PSO-BP and CEEMDANWOA-BP, the MSE of the CEEMDAN-IWOA-BP model decreased by 45.136, 1.508, 2.222 and 0.893 , the RMSE decreased by $36.384,0.276,0.564$, and 0.371 , and the MAPE decreased by $0.385 \%, 0.002 \%, 0.006 \%$, and $0.003 \%$. The results show that the prediction performance of the CEEMDAN-IWOA-BP model is significantly better than the single BP neural network model, the BP neural network model optimized by the traditional WOA, and the BP neural network model optimized by the GA and PSO. It also shows that the TENT chaotic mapping and roulette algorithm introduced in the IWOA can effectively improve the performance of the algorithm, and verify the accuracy and effectiveness of the improved algorithm.

Tab. 1 Evaluation index values of different models

\begin{tabular}{|c|c|c|c|c|}
\hline \multirow{2}{*}{ Predictive model } & \multicolumn{4}{|c|}{ Evaluation index } \\
\hline & $\mathrm{R}^{2}$ & MAPE/\% & $\mathrm{MSE} / \mathrm{mm}^{2}$ & $\mathrm{RMSE} / \mathrm{mm}$ \\
\hline CEEMDAN-BP & 0.992246 & 0.399 & 54.480 & 38.445 \\
\hline CEEMDAN-GA-BP & 0.999950 & 0.016 & 10.852 & 2.337 \\
\hline CEEMDAN-PSO-BP & 0.999947 & 0.020 & 11.566 & 2.625 \\
\hline
\end{tabular}




\begin{tabular}{lcccc}
\hline CEEMDAN-WOA-BP & 0.999953 & 0.017 & 10.237 & 2.432 \\
CEEMDAN-IWOA-BP & 0.999957 & 0.014 & 9.344 & 2.061 \\
\hline
\end{tabular}

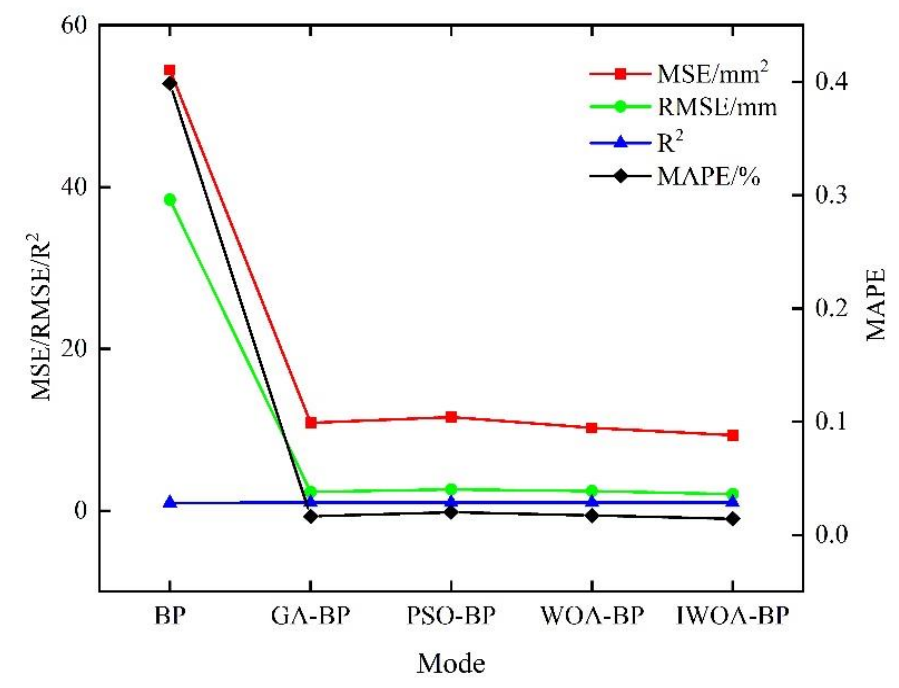

Fig. 9 Forecast errors of different models

\section{Conclusion}

In order to reduce the non-stationarity of the precipitation series and improve the accuracy of precipitation prediction, this paper constructs a hybrid prediction model of CEEMDAN-IWOA-BP. Smoothing by CEEMDAN decomposition, improving the parameter optimization of the WOA and the prediction of the BP neural network, selecting the historical precipitation sequence analysis in Sichuan Province, combining the algorithm in this paper with a single BP model, GA-BP model, PSO-BP model and no improvement Comparison of prediction results of WOA-BP model. The main conclusions are as follows:

The CEEMDAN decomposition preprocesses the non-stationary precipitation series, which solves the defects of modal aliasing and residual white noise, and the decomposition effect is better than EMD and EEMD; in order to improve the 
optimization accuracy and solving ability of the model, Introduce TENT chaotic map and roulette algorithm to improve WOA. Compared with traditional WOA, IWOA's global search capability, optimization accuracy and other solving capabilities are better than traditional WOA; compared with the other four models, the CEEMDAN-IWOABP model has the lowest error value and the highest degree of fit. The error evaluation indicators MAPE, MSE and RMSE are $0.014 \%, 9.344 \mathrm{~mm}^{2}$ and $2.061 \mathrm{~mm}$, respectively, achieving a more accurate precipitation prediction provides a new approach for midand long-term precipitation prediction, and also provides reference value for related prediction research in other regions.

\section{Author Contribution}

All authors contributed to the study conception and design. Writing and editing:

Fuping Liu and Chen Yang; chart editing: Ying Liu; preliminary data collection: Ruixun

Lai. All authors read and approved the final manuscript.

\section{Funding}

This research was supported by the Open Fund of the Key Laboratory of Lower Yellow River Channel and Estuary Regulation (LYRCER202101), Key R \& D and Promotion Projects in Henan Province (202102310261), Program for Young Backbone Teachers in Universities of Henan Province (2020GGJS093).

\section{Data Availability}

Data and materials are available from the corresponding author upon request.

\section{Declarations}

Ethics Approval: Not applicable. 
Consent to Participate: Not applicable.

Consent for Publication: Written informed consent for publication was obtained from all participants.

Competing Interests: The authors declare no competing interests.

\section{References:}

[1] Xia J, LIU CZ, REN G Y. Opportunities and challenges facing the research on the impact of climate change on my country's water resources[J]. Advances in Earth Science, 2011, 26(01): $1-12$.

[2] Yang JH. Research on the planning method of rainwater and flood management and control for the gully settlements on the Loess Plateau in Shanxi and Shanxi[D]. Xi'an University of Architecture and Technology, 2020.

[3] Lei GJ. Research on long-term runoff forecast of Fengman Reservoir based on data fusion[D]. China Institute of Water Resources and Hydropower Research, 2020.

[4] Xu LX, Liang C, Wang SF. Application of Elman model in annual runoff prediction of the upper reaches of the Yellow River[J]. People's Yellow River, 2006(11): 24-25+41.

[5] David ER. Learning representations by back-propagating errors[J]. NATURE, 1986, 323(9): 533-536.

[6] Wang DW, Sun ZW. Power user-side big data analysis and parallel load forecasting[J]. Proceedings of the Chinese Society of Electrical Engineering, 2015, 35(03): 527-537.

[7] Peng Y, Liu DT. Summary of data-driven failure prediction and health management[J]. Chinese Journal of Scientific Instrument, 2014, 35(03): 481-495.

[8] Xie HP, Wu LX, Zheng DZ. China's energy consumption and coal demand forecast in 2025[J]. Journal of China Coal Society, 2019, 44(07): 1949-1960.

[9] Cao JX, Wu JL, Shi W, et al. Analysis and prediction of information dissemination on Sina Weibo[J]. Chinese Journal of Computers, 2014, 37(04): 779-790.

[10] Zhao F, Sun B, Zhang CH. Load forecasting method of combined cooling, heating and power system based on multivariable phase space reconstruction and Kalman filter[J]. Proceedings of the Chinese Society of Electrical Engineering, 2016, 36(02): 399-406 .

[11] Han HR, Yang CF, Song JP. Simulation and prediction of the evolution of land use spatial pattern in Beijing[J]. Advances in Geographical Sciences, 2015, 34(08): 976-986.

[12] Ren FM, Gao H, Liu LL, et al. Research progress and application review of extreme weather and climate event monitoring and prediction[J]. Meteorology, 2014, 40(07): 860-874.

[13] Shen HJ, Luo Y, Zhao ZC, et al. Research on summer precipitation prediction in China based on LSTM network[J]. Climate Change Research Progress, 2020, 16(03): 263-275.

[14] Jia XL, Chen LJ, Gao H, et al. Progress in my country's short-term climate prediction technology[J]. Journal of Applied Meteorology, 2013, 24(06): 641-655.

[15] Sun CZ, Lin XY. Fuzzy weighted Markov model for precipitation prediction and its application [J]. Journal of Systems Engineering, 2003(04): 294-299.

[16] Ahmed M, Gu F, Ball A. Feature Selection and Fault Classification of Reciprocating 
Compressors using a Genetic Algorithm and a Probabilistic Neural Network[J]. Institute of Physics, 2011, 305(1): 1-12.

[17] Bo SY, Won WH, Dong JK, et al. Condition classification of small reciprocating compressor for refrigerators using artificial neural networks and support vector machines[J]. Elsevier Ltd, 2005, 19(2): 371-390.

[18] Wang J, Fei K, Cheng Y. Prediction in precipitation based on improved Adaboost-BP model[J]. Computer Applications, 2017, 37(09): 2689-2693.

[19] Hu BH, Cheng L, Wang XZ, et al. GA-BP prediction model of precipitation in the middle and lower reaches of the Yangtze River during the flood season[J]. Journal of PLA University of Science and Technology (Natural Science Edition), 2016, 17(06): 564-570.

[20] Yu YH, Hu BH, Kong YS, et al. Application of RLS-BP artificial neural network algorithm in precipitation forecast [J]. Meteorological Science, 2004(03): 333-341.

[21] Gou ZJ, Ren JL, Xu M, et al. Application of GA-BP algorithm based on Hadoop in precipitation prediction [J]. Computer System Applications, 2019, 28(09): 140-146.

[22] Qiu YX, Zhang XX, Liu GD. Application of particle swarm optimization optimizate BP in rainfall spatial interpolation[J]. Journal of Yangtze River Scientific Research Institute, 2017, 34(12): 28-32.

[23] Jin TK, Teng J. Hybrid genetic algorithm based on simulated degradation algorithm and its application[J]. Science and Technology Vision, 2013(04): 28-29.

[24] Li C. Improved BP neural network and application based on ant colony algorithm[D]. Northwest Sci-tech University of Agriculture and Forestry, 2018.

[25] Long W, Cai SH, Jiao JJ, et al. Improved whale optimization algorithm for solving large-scale optimization problems[J]. System Engineering Theory and Practice, 2017, 37(11): 2983-2994.

[26] Xu DM, Zhuang WT, Wang WC. Research on monthly runoff prediction based on CEEMDANWD-PSO-LSSVM model[J]. China Rural Water and Hydropower, 2021(08): 54-58+66.

[27] Torres ME, Colominas MA, Schlotthauer GA. A complete ensemble empirical mode decomposition with adaptive noise[C]. 2011 IEEE International Conference on Acoustics, Speech and Signal Processing, Prague, Czech Republic: IEEE, 2011: 4144-4147.

[28] Li J, Li Q. Research on mid-term power load forecasting based on CEEMDAN-permutation entropy and leakage integral ESN[J]. Journal of Electrical Machines and Control, 2015, 19(08): 70-80.

[29] Huang NE, Shen Z, Long SR, et al. The empirical mode decomposition and the Hilbert spectrum for nonlinear and nonstationary time series analysis[J]. Proceedings of the Royal Society A: Mathematical, Physical and Engineering Sciences, 1998(454): 903-995.

[30] Wu Z, Huang N. Ensemble Empirical Mode Decomposition: a Noise-Assisted Data Analysis Method[J]. Advances in Adaptive Data Analysis, 2009(1): 1-41.

[31] Mirjalili S, Lewis A. The whale optimization algorithm[J]. Advances in Engineering Software, 2016, 95: 51-67. 